\title{
Introduction to the Rotating Wave Approximation (RWA): Two Coherent Oscillations
}

\author{
Kazuyuki Fujii \\ International College of Arts and Sciences, Yokohama City University, Yokohama, Japan \\ Email: fujii@yokohama-cu.ac.jp
}

How to cite this paper: Fujii, K. (2017) Introduction to the Rotating Wave Approximation (RWA): Two Coherent Oscillations. Journal of Modern Physics, 8, 2042-2058.

https://doi.org/10.4236/jmp.2017.812124

Received: November 2, 2017

Accepted: November 25, 2017

Published: November 28, 2017

Copyright $\odot 2017$ by author and Scientific Research Publishing Inc.

This work is licensed under the Creative Commons Attribution International License (CC BY 4.0).

http://creativecommons.org/licenses/by/4.0/

\begin{abstract}
In this note, I introduce a mysterious approximation called the rotating wave approximation (RWA) to undergraduates or non-experts who are interested in both Mathematics and Quantum Optics. In Quantum Optics, it plays a very important role in order to obtain an analytic approximate solution of some Schrödinger equation, while it is curious from the mathematical point of view. I explain it carefully with two coherent oscillations for them and expect that they will overcome the problem in the near future.
\end{abstract}

\section{Keywords}

Quantum Optics, Rabi Model, Rotating Wave Approximation, Coherent Oscillation

\section{Introduction}

When undergraduates study Quantum Mechanics, they encounter several approximation methods like the WKB, the Born-Oppenheimer, the Hartree-Fock, etc. In fact, exactly solvable models are very few in Quantum Mechanics, so (many) approximation methods play an important role. ${ }^{1}$

When we study Quantum Optics we again encounter the same situation. We often use a method called the rotating wave approximation (RWA), which means fast oscillating terms (in effective Hamiltonians) removed. Because

$$
\mathrm{e}^{ \pm i n \theta} \Rightarrow \int \mathrm{e}^{ \pm i n \theta} \mathrm{d} \theta=\frac{\mathrm{e}^{ \pm i n \theta}}{ \pm i n} \approx 0
$$

holds if $n$ is large enough. We believe that there is no problem on this

${ }^{1}$ As a text book of Quantum Mechanics I recommend [1] although it is not necessarily standard. 
approximation.

However, in some models slow oscillating terms are removed. Let us show an example. The Euler formula gives

$$
\mathrm{e}^{i \theta}=\cos \theta+i \sin \theta \Rightarrow 2 \cos \theta=\mathrm{e}^{i \theta}+\mathrm{e}^{-i \theta} .
$$

From this, we approximate $2 \cos \theta$ to be

$$
2 \cos \theta=\mathrm{e}^{\mathrm{i} \theta}+\mathrm{e}^{-i \theta}=\mathrm{e}^{\mathrm{i} \theta}\left(1+\mathrm{e}^{-2 i \theta}\right) \approx \mathrm{e}^{\mathrm{i} \theta}
$$

because $\mathrm{e}^{-i \theta}$ goes away from $\mathrm{e}^{i \theta}$ by two times speed, so we neglect this term. In our case $n$ is 2 ! Read the text for more details.

Why is such a "rude" method used? The main reason is to obtain analytic approximate solutions for some important models in Quantum Optics. To the best of our knowledge, we cannot obtain such analytic solutions without RWA.

In this review note, I introduce the rotating wave approximation in details with two models for undergraduates or non-experts. I expect that they will overcome this "high wall" in the near future.

\section{Principles of Quantum Mechanics}

One of targets of the paper is to study and solve the time evolution of a quantum state (which is a superposition of two physical states).

In order to set the stage and to introduce proper notation, let us start with a system of principles of Quantum Mechanics (QM in the following for simplicity). See for example [1] [2] [3] [4]. That is:

\section{System of Principles of QM}

1) Superposition Principle

If $|a\rangle$ and $|b\rangle$ are physical states, then their superposition $\alpha|a\rangle+\beta|b\rangle$ is also a physical state where $\alpha$ and $\beta$ are complex numbers.

2) Schrödinger Equation and Evolution

Time evolution of a physical state proceeds like

$$
|\Psi\rangle \rightarrow U(t)|\Psi\rangle
$$

where $U(t)$ is the unitary evolution operator $\left(U^{\dagger}(t) U(t)=U(t) U^{\dagger}(t)=1\right.$ and $U(0)=1$ ) determined by a Schrödinger Equation.

3) Copenhagen Interpretation ${ }^{2}$

Let $a$ and $b$ be the eigenvalues of an observable $Q$, and $|a\rangle$ and $|b\rangle$ be the normalized eigenstates corresponding to $a$ and $b$. When a state is a superposition $\alpha|a\rangle+\beta|b\rangle$ and we observe the observable $Q$ the state collapses like

$$
\alpha|a\rangle+\beta|b\rangle \rightarrow|a\rangle \text { or } \alpha|a\rangle+\beta|b\rangle \rightarrow|b\rangle
$$

where their collapsing probabilities are $|\alpha|^{2}$ and $|\beta|^{2}$ respectively $\left(|\alpha|^{2}+|\beta|^{2}=1\right)$.

This is called the collapse of the wave function and the probabilistic

${ }^{2}$ There are some researchers who are against this terminology, see for example [4]. However, I don't agree with them because the terminology is nowadays very popular in the world. 
interpretation.

4) Many Particle State and Tensor Product

A multiparticle state can be constructed by the superposition of the Knonecker products of one particle states, which are called the tensor products. For example,

$$
\begin{gathered}
\alpha|a\rangle \otimes|a\rangle+\beta|b\rangle \otimes|b\rangle \equiv \alpha|a, a\rangle+\beta|b, b\rangle \quad\left(|\alpha|^{2}+|\beta|^{2}=1\right) \\
\gamma|a\rangle \otimes|b\rangle+\delta|b\rangle \otimes|a\rangle \equiv \gamma|a, b\rangle+\delta|b, a\rangle \quad\left(|\gamma|^{2}+|\delta|^{2}=1\right)
\end{gathered}
$$

are two particle states.

These will play an essential role in the later sections.

\section{Two-Level System of an Atom}

In order to treat the two-level system of an atom we make a short review of the two-dimensional complex vector space $\mathbf{C}^{2}$ and complex matrix space $M(2 ; \mathbf{C})$ within our necessity. See for example [5].

First we introduce the (famous) Pauli matrices $\left\{\sigma_{1}, \sigma_{2}, \sigma_{3}\right\}$ defined by

$$
\sigma_{1}=\left(\begin{array}{ll}
0 & 1 \\
1 & 0
\end{array}\right), \quad \sigma_{2}=\left(\begin{array}{cc}
0 & -i \\
i & 0
\end{array}\right), \quad \sigma_{3}=\left(\begin{array}{cc}
1 & 0 \\
0 & -1
\end{array}\right)
$$

and set the unit matrx $1_{2}$ by

$$
1_{2}=\left(\begin{array}{ll}
1 & 0 \\
0 & 1
\end{array}\right)
$$

Moreover, we set

$$
\sigma_{+}=\frac{1}{2}\left(\sigma_{1}+i \sigma_{2}\right)=\left(\begin{array}{ll}
0 & 1 \\
0 & 0
\end{array}\right), \quad \sigma_{-}=\frac{1}{2}\left(\sigma_{1}-i \sigma_{2}\right)=\left(\begin{array}{ll}
0 & 0 \\
1 & 0
\end{array}\right) .
$$

Note that $\sigma_{1}=\sigma_{+}+\sigma_{-}$. Then it is easy to see

$$
\left[\frac{1}{2} \sigma_{3}, \sigma_{+}\right]=\sigma_{+},\left[\frac{1}{2} \sigma_{3}, \sigma_{-}\right]=-\sigma_{-}, \quad\left[\sigma_{+}, \sigma_{-}\right]=2 \times \frac{1}{2} \sigma_{3} .
$$

Comment The Pauli matrices $\left\{\sigma_{1}, \sigma_{2}, \sigma_{3}\right\}$ are generators of the (real) Lie algebra $s u(2)$ of the special unitary group $S U(2)$ because of

$$
s u(2)=\left\{i\left(a \sigma_{1}+b \sigma_{2}+c \sigma_{3}\right) \mid a, b, c \in \mathbf{R}\right\}
$$

and $\left\{\sigma_{+}, \sigma_{-}, \frac{1}{2} \sigma_{3}\right\}$ are generators of the (complex) Lie algebra $\operatorname{sl}(2 ; \mathbf{C})$ of the special linear group $S L(2 ; \mathbf{C})$ because of

$$
\operatorname{sl}(2 ; \mathbf{C})=\left\{a \sigma_{+}+b \sigma_{-}+c\left(\sigma_{3} / 2\right) \mid a, b, c \in \mathbf{C}\right\} .
$$

For the sake of readers, we write a Lie diagram of these algebras and groups, see the following Figure 1.

Next, we define $\{|0\rangle,|1\rangle\}$ a basis of $\mathbf{C}^{2}$ by use of the Dirac's notation

$$
|0\rangle=\left(\begin{array}{l}
1 \\
0
\end{array}\right), \quad|1\rangle=\left(\begin{array}{l}
0 \\
1
\end{array}\right) .
$$




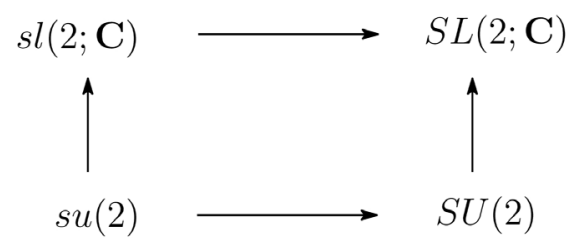

Figure 1. Lie diagram.

Then, since $\sigma_{1}$ satisfies the relation

$$
\sigma_{1}|0\rangle=|1\rangle, \quad \sigma_{1}|1\rangle=|0\rangle
$$

it is called the flip operation.

Note If we define $\left\{\sigma_{+}, \sigma_{-}, \frac{1}{2} \sigma_{3}\right\}$ as above then $\{|0\rangle,|1\rangle\}$ should be chosen

as

$$
|0\rangle=\left(\begin{array}{l}
0 \\
1
\end{array}\right), \quad|1\rangle=\left(\begin{array}{l}
1 \\
0
\end{array}\right)
$$

instead of (3). Because,

$$
\sigma_{-}|0\rangle=0, \quad \sigma_{-}|1\rangle=|0\rangle, \quad \sigma_{+}|0\rangle=|1\rangle .
$$

However, I use the conventional notations in this note.

For the later convenience we calculate the exponential map. For a square matrix $A$ the exponential map is defined by

$$
e^{\lambda A}=\sum_{n=0}^{\infty} \frac{(\lambda A)^{n}}{n !}=\sum_{n=0}^{\infty} \frac{\lambda^{n}}{n !} A^{n}, \quad A^{0}=E,
$$

where $E$ is the unit matrix and $\lambda$ is a constant.

Here, let us calculate $\mathrm{e}^{\mathrm{i} \lambda \sigma_{1}}$ as an example. Noting

$$
\sigma_{1}^{2}=\left(\begin{array}{ll}
0 & 1 \\
1 & 0
\end{array}\right)^{2}=\left(\begin{array}{ll}
1 & 0 \\
0 & 1
\end{array}\right)=1_{2}
$$

we obtain

$$
\begin{aligned}
\mathrm{e}^{i \lambda \sigma_{1}} & =\sum_{n=0}^{\infty} \frac{(i \lambda)^{n} \sigma_{1}^{n}}{n !} \\
& =\sum_{n=0}^{\infty} \frac{(i \lambda)^{2 n}}{(2 n) !} \sigma_{1}^{2 n}+\sum_{n=0}^{\infty} \frac{(i \lambda)^{2 n+1}}{(2 n+1) !} \sigma_{1}^{2 n+1} \\
& =\sum_{n=0}^{\infty} \frac{(-1)^{n} \lambda^{2 n}}{(2 n) !} 1_{2}+i \sum_{n=0}^{\infty} \frac{(-1)^{n} \lambda^{2 n+1}}{(2 n+1) !} \sigma_{1} \\
& =\cos \lambda 1_{2}+i \sin \lambda \sigma_{1} \\
& =\left(\begin{array}{cc}
\cos \lambda & i \sin \lambda \\
i \sin \lambda & \cos \lambda
\end{array}\right) .
\end{aligned}
$$

Exercise Calculate

$$
\mathrm{e}^{\mathrm{i} \lambda \sigma_{2}} \text { and } \mathrm{e}^{\mathrm{i} \lambda \sigma_{3}} \text {. }
$$

We discuss an atom trapped in a cavity and consider only two energy states, 
namely (in our case) the ground state and first excited state. That is, all the remaining states are neglected. This is usually called the two-level approximation. See for example [6] as a general introduction.

We set that energies of the ground state $|0\rangle$ and first excited state $|1\rangle$ are $E_{0}$ and $E_{1}\left(E_{0}<E_{1}\right)$ respectively. Under this approximation the space of all states is two-dimensional, so there is no problem to identify $\{|0\rangle,|1\rangle\}$ with (3).

Then we can write the Hamiltonian in a diagonal form like

$$
H_{0}=\left(\begin{array}{ll}
E_{0} & \\
& E_{1}
\end{array}\right) .
$$

For the later convenience let us transform it. For $\Delta=E_{1}-E_{0}$ the energy difference we have

$$
\begin{aligned}
\left(\begin{array}{ll}
E_{0} & \\
& E_{1}
\end{array}\right) & =\left(\begin{array}{ll}
\frac{E_{0}+E_{1}}{2}-\frac{E_{1}-E_{0}}{2} & \frac{E_{0}+E_{1}}{2}+\frac{E_{1}-E_{0}}{2}
\end{array}\right) \\
& =\frac{E_{0}+E_{1}}{2} 1_{2}-\frac{E_{1}-E_{0}}{2} \sigma_{3} \\
& =\frac{E_{0}+E_{1}}{2} 1_{2}-\frac{\Delta}{2} \sigma_{3} .
\end{aligned}
$$

To this atom we subject LASER (Light Amplification by Stimulated Emission of Radiation) in order to controll it. As an image see the following Figure 2.

In this note we treat Laser as a classical wave for simplicity, which is not so bad as shown in the following. That is, we may set the laser field as

$$
A \cos (\omega t+\phi) \text {. }
$$

By the way, from several experiments we know that an atom subjected by Laser raises an energy level and vice versa. This is expressed by the property of the Pauli matrix $\sigma_{1}$

$$
\sigma_{1}|0\rangle=|1\rangle, \quad \sigma_{1}|1\rangle=|0\rangle,
$$

so we can use $\sigma_{1}$ as the interaction term of the Hamiltonian.

As a result our Hamiltonian (effective Hamiltonian) can be written as

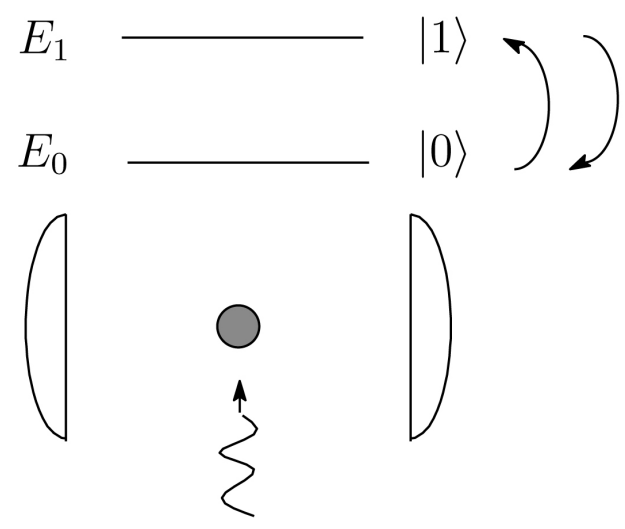

Figure 2. Laser subjecting to an atom. 


$$
H=-\frac{\Delta}{2} \sigma_{3}+2 g \cos (\omega t+\phi) \sigma_{1}=\left(\begin{array}{cc}
-\frac{\Delta}{2} & 2 g \cos (\omega t+\phi) \\
2 g \cos (\omega t+\phi) & \frac{\Delta}{2}
\end{array}\right)
$$

where $g$ is a coupling constant regarding an interaction of between an atom and laser, and $A$ is absorbed in $g(g A \rightarrow g)$. We ignore the scalar term $\frac{E_{0}+E_{1}}{2} 1_{2}$ for simplicity. Note that (6) is semi-classical and time-dependent.

Therefore, our task is to solve the Schrödinger equation

$$
i \hbar \frac{\partial}{\partial t} \Psi=H \Psi
$$

exactly (if possible).

\section{Rotating Wave Approximation}

Unfortunately we cannot solve (7) exactly at the present time. It must be non-integrable although we don't know the proof (see the appendix). Therefore we must apply some approximate method in order to obtain an analytic approximate solution. Now we explain a method called the Rotating Wave Approximation (RWA). Let us recall the Euler formula

$$
\mathrm{e}^{\mathrm{i} \theta}=\cos \theta+i \sin \theta \Rightarrow 2 \cos \theta=\mathrm{e}^{i \theta}+\mathrm{e}^{-i \theta} .
$$

From this we approximate $2 \cos \theta$ to be

$$
2 \cos \theta=\mathrm{e}^{\mathrm{i} \theta}+\mathrm{e}^{-i \theta}=\mathrm{e}^{\mathrm{i} \theta}\left(1+\mathrm{e}^{-2 i \theta}\right) \approx \mathrm{e}^{\mathrm{i} \theta}
$$

because $\mathrm{e}^{-i \theta}$ goes away from $\mathrm{e}^{\mathrm{i} \theta}$ by two times speed, so we neglect this term ! See the following Figure 3. We call this the rotating wave approximation.

Problem In general, fast oscillating terms may be neglected because

$$
\int \mathrm{e}^{ \pm i n \theta} \mathrm{d} \theta=\frac{\mathrm{e}^{ \pm i n \theta}}{ \pm i n} \approx 0
$$

if $n$ is large. Our question is: Is $n=2$ large enough?

By noting that the Hamiltonian should be hermitian, we approximate

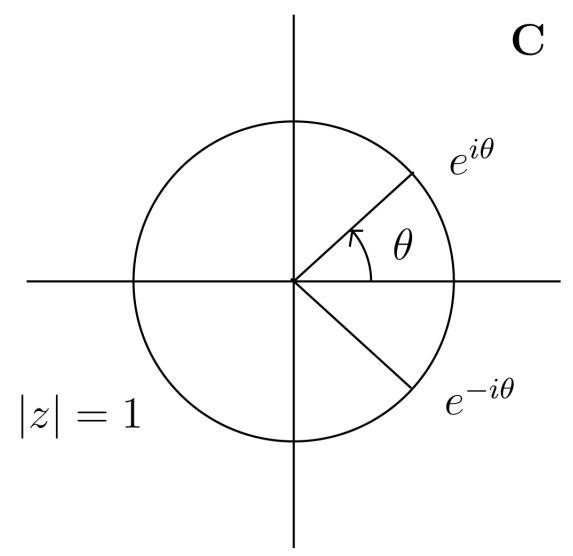

Figure 3. Complex circle. 


$$
2 \cos (\omega t+\phi) \sigma_{1}=\left(\begin{array}{cc}
0 & 2 \cos (\omega t+\phi) \\
2 \cos (\omega t+\phi) & 0
\end{array}\right) \approx\left(\begin{array}{cc}
0 & \mathrm{e}^{i(\omega t+\phi)} \\
\mathrm{e}^{-i(\omega t+\phi)} & 0
\end{array}\right),
$$

by use of (8), so (6) is reduced to

$$
\tilde{H}=\left(\begin{array}{cc}
-\frac{\Delta}{2} & g \mathrm{e}^{i(\omega t+\phi)} \\
g \mathrm{e}^{-i(\omega t+\phi)} & \frac{\Delta}{2}
\end{array}\right) .
$$

As a result our modified task is to solve the Schrödinger equation

$$
i \hbar \frac{\partial}{\partial t} \Psi=\tilde{H} \Psi
$$

exactly. Mysteriously enough, this equation can be solved easily.

Note For the latter convenience let us rewrite the method with formal notations:

$$
\begin{aligned}
2 \cos \theta \sigma_{1} & =\sigma_{1} \otimes 2 \cos \theta=\left(\sigma_{+}+\sigma_{-}\right) \otimes\left(\mathrm{e}^{\mathrm{i} \theta}+\mathrm{e}^{-i \theta}\right) \\
& =\sigma_{+} \otimes \mathrm{e}^{\mathrm{i} \theta}+\sigma_{+} \otimes \mathrm{e}^{-i \theta}+\sigma_{-} \otimes \mathrm{e}^{i \theta}+\sigma_{-} \otimes \mathrm{e}^{-i \theta} \rightarrow \sigma_{+} \otimes \mathrm{e}^{\mathrm{i} \theta}+\sigma_{-} \otimes \mathrm{e}^{-i \theta} .
\end{aligned}
$$

In order to solve (10) we set $\hbar=1$ for simplicity. From (9) it is easy to see

$$
\left(\begin{array}{cc}
-\frac{\Delta}{2} & g \mathrm{e}^{i(\omega t+\phi)} \\
g \mathrm{e}^{-i(\omega t+\phi)} & \frac{\Delta}{2}
\end{array}\right)=\left(\begin{array}{cc}
\mathrm{e}^{i \frac{\omega t+\phi}{2}} & \\
& \mathrm{e}^{-i \frac{\omega t+\phi}{2}}
\end{array}\right)\left(\begin{array}{cc}
-\frac{\Delta}{2} & g \\
g & \frac{\Delta}{2}
\end{array}\right)\left(\begin{array}{ll}
\mathrm{e}^{-i \frac{\omega t+\phi}{2}} & \\
& \mathrm{e}^{i \frac{\omega t+\phi}{2}}
\end{array}\right),
$$

so we transform the wave function $\Psi$ in (10) into

$$
\Phi=\left(\begin{array}{ll}
\mathrm{e}^{-i \frac{\omega t+\phi}{2}} & \\
& \mathrm{e}^{i \frac{\omega t+\phi}{2}}
\end{array}\right) \Psi \Leftrightarrow \Psi=\left(\begin{array}{ll}
\mathrm{e}^{i \frac{\omega t+\phi}{2}} & \\
& \mathrm{e}^{-i \frac{\omega t+\phi}{2}}
\end{array}\right) \Phi .
$$

Then the Schrödinger Equation (10) becomes

$$
i \frac{\partial}{\partial t} \Phi=\left(\begin{array}{cc}
-\frac{\Delta-\omega}{2} & g \\
g & \frac{\Delta-\omega}{2}
\end{array}\right) \Phi
$$

by a straightforward calculation.

Here we set the resonance condition

$$
\Delta=\omega \quad\left(\Leftrightarrow E_{1}-E_{0}=\hbar \omega \text { precisely }\right)
$$

Namely, we subject the laser field with $\omega$ equal to the energy difference $\Delta$. See the following Figure 4.

Then (12) becomes

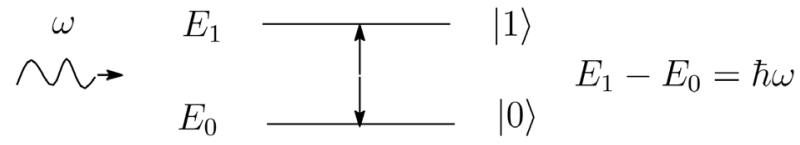

Figure 4. Laser and energy difference. 


$$
i \frac{\partial}{\partial t} \Phi=\left(\begin{array}{ll}
0 & g \\
g & 0
\end{array}\right) \Phi=g \sigma_{1} \Phi
$$

and we have only to solve the equation

$$
\frac{\partial}{\partial t} \Phi=-i g \sigma_{1} \Phi
$$

By $(4)(\lambda=-g t)$ the solution is

$$
\Phi(t)=\mathrm{e}^{-i g t \sigma_{1}} \Phi(0)=\left(\begin{array}{cc}
\cos (g t) & -i \sin (g t) \\
-i \sin (g t) & \cos (g t)
\end{array}\right) \Phi(0),
$$

and coming back to $\Psi$ (from $\Phi$ ) we obtain

$$
\begin{aligned}
\Psi(t) & =\left(\begin{array}{cc}
\mathrm{e}^{i \frac{\omega t+\phi}{2}} & \\
& \mathrm{e}^{-i \frac{\omega t+\phi}{2}}
\end{array}\right)\left(\begin{array}{cc}
\cos (g t) & -i \sin (g t) \\
-i \sin (g t) & \cos (g t)
\end{array}\right) \Psi(0) \\
& =\mathrm{e}^{i \frac{\omega t+\phi}{2}}\left(\begin{array}{cc}
1 & \\
\mathrm{e}^{-i(\omega t+\phi)}
\end{array}\right)\left(\begin{array}{cc}
\cos (g t) & -i \sin (g t) \\
-i \sin (g t) & \cos (g t)
\end{array}\right) \Psi(0) \\
& =\left(\begin{array}{cc}
\cos (g t) & -i \sin (g t) \\
-i \mathrm{e}^{-i(\omega t+\phi)} \sin (g t) & \mathrm{e}^{-i(\omega t+\phi)} \cos (g t)
\end{array}\right) \Psi(0)
\end{aligned}
$$

by (11) $(\Psi(0)=\Phi(0))$ because the total phase $\mathrm{e}^{i \frac{i t+\phi}{2}}$ can be neglected in Quantum Mechanics.

As an initial condition, if we choose

$$
\Psi(0)=|0\rangle=\left(\begin{array}{l}
1 \\
0
\end{array}\right)
$$

we have

$$
\begin{aligned}
\Psi(t) & =\left(\begin{array}{c}
\cos (g t) \\
-i \mathrm{e}^{-i(\omega t+\phi)} \sin (g t)
\end{array}\right)=\left(\begin{array}{c}
\cos (g t) \\
\mathrm{e}^{-i(\omega t+\phi+\pi / 2)} \sin (g t)
\end{array}\right) \\
& =\cos (g t)|0\rangle+\mathrm{e}^{-i(\omega t+\phi+\pi / 2)} \sin (g t)|1\rangle
\end{aligned}
$$

by (3). That is, $\Psi(t)$ oscillates between the two states $|0\rangle$ and $|1\rangle$. This is called the coherent oscillation or the Rabi oscillation, which plays an essential role in Quantum Optics.

Concerning an application of this oscillation to Quantum Computation see for example [4].

Problem Our real target is to solve the Schrödinger equation

$$
i \hbar \frac{\partial}{\partial t} \Psi=H \Psi
$$

with

$$
H=H(t)=\left(\begin{array}{cc}
-\frac{\Delta}{2} & 2 g \cos (\omega t+\phi) \\
2 g \cos (\omega t+\phi) & \frac{\Delta}{2}
\end{array}\right) .
$$


Present a new idea and solve the equation.

\section{Quantum Rabi Model}

In this section, we discuss the quantum Rabi model whose Hamiltonian is given by

$$
\begin{aligned}
H & =\frac{\Omega}{2} \sigma_{3} \otimes \mathbf{1}+\omega 1_{2} \otimes a^{\dagger} a+g \sigma_{1} \otimes\left(a+a^{\dagger}\right) \\
& =\frac{\Omega}{2} \sigma_{3} \otimes 1+\omega 1_{2} \otimes N+g\left(\sigma_{+}+\sigma_{-}\right) \otimes\left(a+a^{\dagger}\right)
\end{aligned}
$$

where $\mathbf{1}$ is the identity operator on the Fock space $\mathcal{F}$ generated by the Heisenberg algebra $\left\{a, a^{\dagger}, N \equiv a^{\dagger} a\right\}$, and $\Omega$ and $\omega$ are constant, and $g$ is a coupling constant. As a general introduction to this model see for example see [6].

Let us recall the fundamental relations of the Heisenberg algebra

$$
\left[N, a^{\dagger}\right]=a^{\dagger}, \quad[N, a]=-a,\left[a, a^{\dagger}\right]=\mathbf{1} .
$$

Here, the Fock space $\mathcal{F}$ is a Hilbert space over $\mathbf{C}$ given by

$$
\mathcal{F}=\operatorname{Vect}_{\mathbf{c}}\{|0\rangle,|1\rangle, \cdots,|n\rangle, \cdots\}
$$

where $|0\rangle$ is the vacuum $(a|0\rangle=0)$ and $|n\rangle$ is given by

$$
|n\rangle=\frac{\left(a^{\dagger}\right)^{n}}{\sqrt{n !}}|0\rangle \quad \text { for } n \geq 0
$$

On this space, the operators (=infinite dimensional matrices) $a^{\dagger}, a$ and $N$ are represented as

$$
\begin{aligned}
& a=\left(\begin{array}{ccccc}
0 & 1 & & & \\
& 0 & \sqrt{2} & & \\
& 0 & \sqrt{3} & \\
& & 0 & \ddots \\
& & & & \ddots
\end{array}\right), a^{\dagger}=\left(\begin{array}{ccccc}
0 & & & & \\
1 & 0 & & & \\
& \sqrt{2} & 0 & & \\
& & \sqrt{3} & 0 & \\
& & & \ddots & \ddots
\end{array}\right), \\
& N=a^{\dagger} a=\left(\begin{array}{ccccc}
0 & & & & \\
& 1 & & & \\
& & 2 & & \\
& & 3 & \\
& & & \ddots
\end{array}\right)
\end{aligned}
$$

by use of (17).

Note We can add a phase to $\left\{a, a^{\dagger}\right\}$ like

$$
b=\mathrm{e}^{i \theta} a, \quad b^{\dagger}=\mathrm{e}^{-i \theta} a^{\dagger}, \quad N=b^{\dagger} b=a^{\dagger} a
$$

where $\theta$ is constant. Then we have another Heisenberg algebra

$$
\left[N, b^{\dagger}\right]=b^{\dagger}, \quad[N, b]=-b, \quad\left[b, b^{\dagger}\right]=\mathbf{1} .
$$

Again, we would like to solve Schrödinger equation ( $\hbar=1$ for simplicity) 


$$
i \frac{\partial}{\partial t}|\Psi\rangle=H|\Psi\rangle=\left\{\frac{\Omega}{2} \sigma_{3} \otimes \mathbf{1}+\omega 1_{2} \otimes N+g\left(\sigma_{+}+\sigma_{-}\right) \otimes\left(a+a^{\dagger}\right)\right\}|\Psi\rangle
$$

exactly. To the best of our knowledge the exact solution has not been known, so we must use some approximation in order to obatin an analytic solution.

Since

$$
\left(\sigma_{+}+\sigma_{-}\right) \otimes\left(a+a^{\dagger}\right)=\sigma_{+} \otimes a+\sigma_{+} \otimes a^{\dagger}+\sigma_{-} \otimes a+\sigma_{-} \otimes a^{\dagger},
$$

we neglect the middle terms $\sigma_{+} \otimes a^{\dagger}+\sigma_{-} \otimes a$ and set

$$
\tilde{H}=\frac{\Omega}{2} \sigma_{3} \otimes \mathbf{1}+\omega 1_{2} \otimes N+g\left(\sigma_{+} \otimes a+\sigma_{-} \otimes a^{\dagger}\right) .
$$

This is called the rotating wave approximation and the resultant Hamiltonian is called the Jaynes-Cummings one ${ }^{3}$, [7].

Therefore, our modified task is to solve the Schrödinger equation

$$
i \frac{\partial}{\partial t}|\Psi\rangle=\tilde{H}|\Psi\rangle=\left\{\frac{\Omega}{2} \sigma_{3} \otimes \mathbf{1}+\omega 1_{2} \otimes N+g\left(\sigma_{+} \otimes a+\sigma_{-} \otimes a^{\dagger}\right)\right\}|\Psi\rangle
$$

exactly. Mysteriously enough, to solve the equation is very easy.

For a unitary operator $U=U(t)$ we set

$$
|\Phi\rangle=U|\Psi\rangle \text {. }
$$

Then it is easy to see

$$
i \frac{\partial}{\partial t}|\Phi\rangle=\left(U \tilde{H} U^{-1}+i \frac{\partial U}{\partial t} U^{-1}\right)|\Phi\rangle
$$

by (21). If we choose $U$ as

$$
U(t)=\mathrm{e}^{i t \frac{\omega}{2} \sigma_{3}} \otimes \mathrm{e}^{i t \omega N}=\left(\begin{array}{ll}
\mathrm{e}^{i t\left(\omega N+\frac{\omega}{2}\right)} & \\
& \left.\mathrm{e}^{i t\left(\omega N-\frac{\omega}{2}\right)}\right)
\end{array}\right.
$$

(we use $\frac{\omega}{2}$ in place of $\frac{\omega}{2} \mathbf{1}$ for simplicity), a straightforward calculation gives

$$
U \tilde{H} U^{-1}+i \frac{\partial U}{\partial t} U^{-1}=\left(\begin{array}{cc}
\frac{\Omega-\omega}{2} & g a \\
g a^{\dagger} & -\frac{\Omega-\omega}{2}
\end{array}\right)
$$

and we have a simple equation

$$
i \frac{\partial}{\partial t}|\Phi\rangle=\left(\begin{array}{cc}
\frac{\Omega-\omega}{2} & g a \\
g a^{\dagger} & -\frac{\Omega-\omega}{2}
\end{array}\right)|\Phi\rangle .
$$

Note that in the process of calculation we have used the relations

$$
\mathrm{e}^{i t \omega N} a \mathrm{e}^{-i t \omega N}=\mathrm{e}^{-i t \omega} a, \quad \mathrm{e}^{i t \omega N} a^{\dagger} \mathrm{e}^{-i t \omega N}=\mathrm{e}^{i t \omega} a^{\dagger} .
$$

The proof is easy by use of the formula

${ }^{3}$ In [6], it is called the Jaynes-Cummings-Paul one. 


$$
\mathrm{e}^{X} A \mathrm{e}^{-X}=A+[X, A]+\frac{1}{2 !}[X,[X, A]]+\frac{1}{3 !}[X,[X,[X, A]]]+\cdots
$$

for square matrices $X, A$ and (17).

Here we set the resonance condition

$$
\Omega=\omega,
$$

then (23) becomes

$$
i \frac{\partial}{\partial t}|\Phi\rangle=\left(g^{\dagger} \quad g a\right)|\Phi\rangle=g\left(a_{a^{\dagger}}^{a}\right)|\Phi\rangle .
$$

Let us solve this equation. By setting

$$
A=\left(\begin{array}{ll} 
& a \\
a^{\dagger} &
\end{array}\right)
$$

we calculate the term $\mathrm{e}^{-i g t A}$. Noting

$$
A^{2}=\left(\begin{array}{cc}
a a^{\dagger} & \\
& a^{\dagger} a
\end{array}\right)=\left(\begin{array}{cc}
N+1 & \\
& N
\end{array}\right) \quad\left(\Leftarrow\left[a, a^{\dagger}\right]=1\right)
$$

we have

$$
\begin{aligned}
& \mathrm{e}^{-i g t A}=\sum_{n=0}^{\infty} \frac{(-i g t)^{n} A^{n}}{n !}=\sum_{n=0}^{\infty} \frac{(-i g t)^{2 n}}{(2 n) !} A^{2 n}+\sum_{n=0}^{\infty} \frac{(-i g t)^{2 n+1}}{(2 n+1) !} A^{2 n+1} \\
& =\sum_{n=0}^{\infty} \frac{(-1)^{n}(g t)^{2 n}}{(2 n) !}\left(\begin{array}{r}
(N+1)^{n} \\
N^{n}
\end{array}\right)-i \sum_{n=0}^{\infty} \frac{(-1)^{n}(g t)^{2 n+1}}{(2 n+1) !}\left(\begin{array}{c}
(N+1)^{n} a \\
N^{n} a^{\dagger}
\end{array}\right) \\
& =\left(\begin{array}{c}
\cos (\sqrt{N+1} g t) \\
\cos (\sqrt{N} g t)
\end{array}\right)-i\left(\begin{array}{c}
\left.\frac{1}{\sqrt{N+1}} \sin (\sqrt{N+1} g t) a\right) \\
\sin (\sqrt{N} g t) a^{\dagger}
\end{array}\right) \\
& =\left(\begin{array}{cc}
\cos (\sqrt{N+1} g t) & -i \frac{1}{\sqrt{N+1}} \sin (\sqrt{N+1} g t) a \\
-i \frac{1}{\sqrt{N}} \sin (\sqrt{N} g t) a^{\dagger} & \cos (\sqrt{N} g t)
\end{array}\right) .
\end{aligned}
$$

Therefore, the solution is given by

$$
|\Phi(t)\rangle=\left(\begin{array}{cc}
\cos (\sqrt{N+1} g t) & -i \frac{1}{\sqrt{N+1}} \sin (\sqrt{N+1} g t) a \\
-i \frac{1}{\sqrt{N}} \sin (\sqrt{N} g t) a^{\dagger} & \cos (\sqrt{N} g t)
\end{array}\right)|\Phi(0)\rangle
$$

and coming back to $|\Psi\rangle$ (from $|\Phi\rangle$ ) we finally obtain

$$
\begin{aligned}
& |\Psi(t)\rangle=\left(\begin{array}{ll}
\mathrm{e}^{i t\left(\omega N+\frac{\omega}{2}\right)} & \\
& \left.\mathrm{e}^{i t\left(\omega N-\frac{\omega}{2}\right)}\right)
\end{array}\right. \\
& \times\left(\begin{array}{cc}
\cos (\sqrt{N+1} g t) & -i \frac{1}{\sqrt{N+1}} \sin (\sqrt{N+1} g t) a \\
-i \frac{1}{\sqrt{N}} \sin (\sqrt{N} g t) a^{\dagger} & \cos (\sqrt{N} g t)
\end{array}\right)|\Psi(0)\rangle
\end{aligned}
$$


where $|\Psi(0)\rangle=|\Phi(0)\rangle$.

As an initial condition, if we choose a simple state

$$
|\Psi(0)\rangle=\left(\begin{array}{l}
1 \\
0
\end{array}\right) \otimes|0\rangle=\left(\begin{array}{c}
|0\rangle \\
0
\end{array}\right)
$$

(see Appendix B or more generally [5] about the tensor product) we have

$$
|\Psi(t)\rangle=\left(\begin{array}{c}
\mathrm{e}^{i t \frac{\omega}{2}} \cos (g t)|0\rangle \\
-i \mathrm{e}^{i \frac{\omega}{2}} \sin (g t)|1\rangle
\end{array}\right)
$$

because $a|0\rangle=0$ and $a^{\dagger}|0\rangle=|1\rangle$, or

$$
|\Psi(t)\rangle=\cos (g t)\left(\begin{array}{l}
1 \\
0
\end{array}\right) \otimes|0\rangle+\mathrm{e}^{-i \frac{\pi}{2}} \sin (g t)\left(\begin{array}{l}
0 \\
1
\end{array}\right) \otimes|1\rangle
$$

where the total phase $\mathrm{e}^{i t \frac{\omega}{2}}$ has been removed.

Problem Our real target is to solve the Schrödinger equation

$$
i \hbar \frac{\partial}{\partial t}|\Psi\rangle=H|\Psi\rangle
$$

with

$$
H=H(t)=\frac{\Omega}{2} \sigma_{3} \otimes \mathbf{1}+\omega 1_{2} \otimes N+g\left(\sigma_{+}+\sigma_{-}\right) \otimes\left(a+a^{\dagger}\right)
$$

Present a new idea and solve the equation.

As a developed version of the Jaynes-Cummings model see for example [8] and [9].

\section{Concluding Remarks}

In this note, I introduced the rotating wave approximation which plays an important role in Quantum Optics with two examples. The problem is that the method is used even in a subtle case. As far as I know, it is very hard to obtain an analytic approximate solution without RWA.

I don't know the reason why it is so. However, such a "temporary" method must be overcome in the near future. I expect that young researchers will attack and overcome this problem.

Concerning a recent criticism to RWA see [10] and its references, and concerning recent applications to the dynamical Casimir effect see [11] [12] [13] [14] ([13] and [14] are highly recommended).

\section{References}

[1] Green, H.S. (1965) Matrix Mechanics. P. Noordhoff Ltd., Groningen.

[2] Dirac, P. (1958) The Principles of Quantum Mechanics. 4th Edition, Oxford University Press.

[3] Peres, A. (1995) Quantum Theory: Concepts and Methods. Kluwer Academic Publishers, Dordrecht.

[4] Hosoya, A. (1999) Lectures on Quantum Computation (in Japanese), SGC Library 
4. Saiensu-sha Co., Ltd. Publishers, Tokyo.

[5] Fujii, K., et al. (2010) Treasure Box of Mathematical Sciences (in Japanese). Yusei-sha, Tokyo.

[6] Schleich, W.P. (2001) Quantum Optics in Phase Space. WILEY-CH, Berlin. https://doi.org/10.1002/3527602976

[7] Jaynes, E.T. and Cummings, F.W. (1963) Comparison of Quantum and Semiclassical Radiation Theories with Applications to the Beam Maser. Proceedings of the IEEE, 51, 89-109. https://doi.org/10.1109/PROC.1963.1664

[8] Fujii, K. and Suzuki, T. (2011) An Approximate Solution of the Jaynes-Cummings Model with Dissipation. International Journal of Geometric Methods in Modern Physics, 8, 1799-1814. arXiv: 1103.0329[math-ph]. https://doi.org/10.1142/S0219887811005944

[9] Fujii, K. and Suzuki, T. (2012) An Approximate Solution of the Jaynes-Cummings Model with Dissipation II: Another Approach. International Journal of Geometric Methods in Modern Physics, 9, Article ID: 1250036, arXiv: 1108.2322[math-ph]. https://doi.org/10.1142/S0219887812500363

[10] Larson, J. (2013) On the Rotating Wave Approximation in the Adiabatic Limit. Physica Scripta, 2013, Article ID: 014040, arXiv: 1208.1891 [quant-ph]. https://doi.org/10.1088/0031-8949/2013/T153/014040

[11] Law, C.K. (1994) Effective Hamiltonian for the Radiation in a Cavity with a Moving Mirror and a Time-Varying Dielectric Medium. Physical Review A, 49, 309. https://doi.org/10.1103/PhysRevA.49.433

[12] Dodonov, A.V. and Dodonov, V.V. (2012) Approximate Analytical Results on the Cavity Casimir Effect in the Presence of a Two-Level Atom. Physical Review A, 85, Article ID: 063804, arXiv: 1112.0523[quant-ph]. https://doi.org/10.1103/PhysRevA.85.015805

[13] Fujii, K. and Suzuki, T. (2013) An Approximate Solution of the Dynamical Casimir Effect in a Cavity with a Two-Level Atom. International Journal of Geometric Methods in Modern Physics, 10, Article ID: 1350035, arXiv: 1209.5133[quant-ph]. https://doi.org/10.1142/S0219887813500357

[14] Fujii, K. and Suzuki, T. (2014) Rotating Wave Approximation of the Law's Effective Hamiltonian on the Dynamical Casimir Effect. International Journal of Geometric Methods in Modern Physics, 11, 1450003, arXiv: 1209.5133[quant-ph]. https://doi.org/10.1142/S0219887814500030

[15] Barenco, A. (1996) Quantum Physics and Computers. Contemporary Physics, 37, 375-389, arXiv: quant-ph/9612014. https://doi.org/10.1080/00107519608217543 


\section{Appendix}

\section{[A] Another Approach}

Let us give another approach to the derivation (4), which may be smart enough. It is easy to see the diagonal form

$$
\sigma_{1}=W \sigma_{3} W^{-1}
$$

where $W$ is the Walsh-Hadamard matrix (operation) given by

$$
W=\frac{1}{\sqrt{2}}\left(\begin{array}{cc}
1 & 1 \\
1 & -1
\end{array}\right) \in O(2) .
$$

Note that

$$
W^{2}=1_{2} \Rightarrow W=W^{-1}
$$

Then we obtain

$$
\begin{aligned}
\mathrm{e}^{i \lambda \sigma_{1}} & =\mathrm{e}^{i \lambda W \sigma_{3} W^{-1}}=W \mathrm{e}^{i \lambda \sigma_{3}} W^{-1} \\
& =\frac{1}{2}\left(\begin{array}{cc}
1 & 1 \\
1 & -1
\end{array}\right)\left(\begin{array}{cc}
\mathrm{e}^{i \lambda} & \\
\mathrm{e}^{-i \lambda}
\end{array}\right)\left(\begin{array}{cc}
1 & 1 \\
1 & -1
\end{array}\right) \\
& =\left(\begin{array}{cc}
\frac{\mathrm{e}^{i \lambda}+\mathrm{e}^{-i \lambda}}{2} & \frac{\mathrm{e}^{i \lambda}-\mathrm{e}^{-i \lambda}}{2} \\
\frac{\mathrm{e}^{i \lambda}-\mathrm{e}^{-i \lambda}}{2} & \frac{\mathrm{e}^{i \lambda}+\mathrm{e}^{-i \lambda}}{2}
\end{array}\right) \\
& =\left(\begin{array}{cc}
\cos \lambda & i \sin \lambda \\
i \sin \lambda & \cos \lambda
\end{array}\right) .
\end{aligned}
$$

Readers should remark that the Walsh-Hadamard matrix $W$ plays an essential role in Quantum Computation. See for example [15] (note: $W \rightarrow U_{A}$ in this paper).

\section{[B] Tensor Product}

Let us give a brief introduction to the tensor product of matrices. For $A=\left(a_{i j}\right) \in M(m ; \mathbf{C})$ and $B=\left(b_{i j}\right) \in M(n ; \mathbf{C})$ the tensor product is defined by

$$
A \otimes B=\left(a_{i j}\right) \otimes B=\left(a_{i j} B\right) \in M(m n ; \mathbf{C}) .
$$

Precisely, in case of $m=2$ and $n=3$

$$
\begin{aligned}
A \otimes B & =\left(\begin{array}{ll}
a_{11} & a_{12} \\
a_{21} & a_{22}
\end{array}\right) \otimes B=\left(\begin{array}{llll}
a_{11} B & a_{12} B \\
a_{21} B & a_{22} B
\end{array}\right) \\
& =\left(\begin{array}{llllll}
a_{11} b_{11} & a_{11} b_{12} & a_{11} b_{13} & a_{12} b_{11} & a_{12} b_{12} & a_{12} b_{13} \\
a_{11} b_{21} & a_{11} b_{22} & a_{11} b_{23} & a_{12} b_{21} & a_{12} b_{22} & a_{12} b_{23} \\
a_{11} b_{31} & a_{11} b_{32} & a_{11} b_{33} & a_{12} b_{31} & a_{12} b_{32} & a_{12} b_{33} \\
a_{21} b_{11} & a_{21} b_{12} & a_{21} b_{13} & a_{22} b_{11} & a_{22} b_{12} & a_{22} b_{13} \\
a_{21} b_{21} & a_{21} b_{22} & a_{21} b_{23} & a_{22} b_{21} & a_{22} b_{22} & a_{22} b_{23} \\
a_{21} b_{31} & a_{21} b_{32} & a_{21} b_{33} & a_{22} b_{31} & a_{22} b_{32} & a_{22} b_{33}
\end{array}\right) .
\end{aligned}
$$

When I was a young student in Japan this product was called the Kronecker one. Nowadays, it is called the tensor product in a unified manner, which may be better. 
Note that

$$
1_{2} \otimes B=\left(\begin{array}{llllll}
b_{11} & b_{12} & b_{13} & & & \\
b_{21} & b_{22} & b_{23} & & & \\
b_{31} & b_{32} & b_{33} & & & \\
& & & b_{11} & b_{12} & b_{13} \\
& & & b_{21} & b_{22} & b_{23} \\
& & & b_{31} & b_{32} & b_{33}
\end{array}\right),
$$

while

$$
B \otimes 1_{2}=\left(\begin{array}{llllll}
b_{11} & & b_{12} & & b_{13} & \\
& b_{11} & & b_{12} & & b_{13} \\
b_{21} & & b_{22} & & b_{23} & \\
& b_{21} & & b_{22} & & b_{23} \\
b_{31} & & b_{32} & & b_{33} & \\
& b_{31} & & b_{32} & & b_{33}
\end{array}\right) .
$$

The blanks in the matrices above are of course zero.

Readers should recognize the difference. See for example [5] for more details.

\section{[C] Beyond the RWA}

Let us try to solve the Equation (7). For the purpose it is convenient to assume a form for some solution

$$
\Psi(t)=\mathrm{e}^{-i F(t) \sigma_{+}} \mathrm{e}^{-i G(t) \tau_{3}} \mathrm{e}^{-i H(t) \sigma_{-}} \Psi(0), \quad F(0)=G(0)=H(0)=0
$$

where we set $\tau_{3}=(1 / 2) \sigma_{3}$ for simplicity. Note that this form called the disentangling form (a kind of Gauss decomposition of some matrices) is very popular in Quantum Physics.

By setting $\hbar=1$ for simplicity in (7) we must calculate

$$
\begin{aligned}
i \frac{\partial}{\partial t} \Psi & =\left\{-\Delta \tau_{3}+2 g \cos (\omega t+\phi)\left(\sigma_{+}+\sigma_{-}\right)\right\} \Psi \\
& =\left\{2 g \cos (\omega t+\phi) \sigma_{+}-\Delta \tau_{3}+2 g \cos (\omega t+\phi) \sigma_{-}\right\} \Psi
\end{aligned}
$$

where $\tau_{3}=(1 / 2) \sigma_{3}$.

Then we have

$$
\begin{aligned}
& i \frac{\partial}{\partial t} \Psi=i \frac{\partial}{\partial t}\left\{\mathrm{e}^{-i F(t) \sigma_{+}} \mathrm{e}^{-i G(t) \tau_{3}} \mathrm{e}^{-i H(t) \sigma_{-}}\right\} \Psi(0) \\
& =\left\{\dot{F}(t) \sigma_{+}+\dot{G}(t) \mathrm{e}^{-i F(t) \sigma_{+}} \tau_{3} \mathrm{e}^{i F(t) \sigma_{+}}+\dot{H}(t) \mathrm{e}^{-i F(t) \sigma_{+}} \mathrm{e}^{-i G(t) \tau_{3}} \sigma_{-} \mathrm{e}^{i G(t) \tau_{3}} \mathrm{e}^{i F(t) \sigma_{+}}\right\} \Psi .
\end{aligned}
$$

From (2)

$$
\left[\tau_{3}, \sigma_{+}\right]=\sigma_{+}, \quad\left[\tau_{3}, \sigma_{-}\right]=-\sigma_{-}, \quad\left[\sigma_{+}, \sigma_{-}\right]=2 \tau_{3}
$$

and the formula (24) it is easy to see

$$
\begin{gathered}
\mathrm{e}^{-i F(t) \sigma_{+}} \tau_{3} \mathrm{e}^{i F(t) \sigma_{+}}=\tau_{3}+i F(t) \sigma_{+}, \\
\mathrm{e}^{-i G(t) \tau_{3}} \sigma_{-} \mathrm{e}^{i G(t) \tau_{3}}=(1+i G(t)) \sigma_{-}, \\
\mathrm{e}^{-i F(t) \sigma_{+}} \sigma_{-} \mathrm{e}^{i F(t) \sigma_{+}}=\sigma_{-}-2 i F(t) \tau_{3}+F(t)^{2} \sigma_{+} .
\end{gathered}
$$

Therefore 


$$
\begin{aligned}
i \frac{\partial}{\partial t} \Psi= & \left\{\dot{F}(t) \sigma_{+}+\dot{G}(t)\left(\tau_{3}+i F(t) \sigma_{+}\right)\right. \\
& \left.+\dot{H}(t)(1+i G(t))\left(\sigma_{-}-2 i F(t) \tau_{3}+F(t)^{2} \sigma_{+}\right)\right\} \Psi \\
= & {\left[\left\{\dot{F}(t)+i F(t) \dot{G}(t)+(1+i G(t)) F(t)^{2} \dot{H}(t)\right\} \sigma_{+}\right.} \\
& \left.+\{\dot{G}(t)-2 i(1+i G(t)) F(t) \dot{H}(t)\} \tau_{3}+(1+i G(t)) \dot{H}(t) \sigma_{-}\right] \Psi .
\end{aligned}
$$

By comparing two equations above we obtain a system of differential equations

$$
\left\{\begin{array}{l}
\dot{F}(t)+i F(t) \dot{G}(t)+(1+i G(t)) F(t)^{2} \dot{H}(t)=2 g \cos (\omega t+\phi), \\
\dot{G}(t)-2 i(1+i G(t)) F(t) \dot{H}(t)=-\Delta, \\
(1+i G(t)) \dot{H}(t)=2 g \cos (\omega t+\phi) .
\end{array}\right.
$$

By deforming them we have

$$
\left\{\begin{array}{l}
\dot{F}(t)-i \Delta F(t)-2 g \cos (\omega t+\phi) F(t)^{2}=2 g \cos (\omega t+\phi), \\
\dot{G}(t)-4 i g \cos (\omega t+\phi) F(t)=-\Delta, \\
(1+i G(t)) \dot{H}(t)=2 g \cos (\omega t+\phi) .
\end{array}\right.
$$

This is a simple exercise for young students.

If we can solve the first equation then we obtain solutions like

$$
F(t) \Rightarrow G(t) \Rightarrow H(t) \text {. }
$$

The first equation

$$
\dot{F}(t)-2 g \cos (\omega t+\phi)-i \Delta F(t)-2 g \cos (\omega t+\phi) F(t)^{2}=0
$$

is a (famous) Riccati equation of general type. Unfortunately, we don't know how to solve it explicitly at the present time.

\section{[D] Full Calculation}

Let us give the full calculation to the Equation (23). We set

$$
B=\left(\begin{array}{cc}
\frac{\Omega-\omega}{2} & g a \\
g a^{\dagger} & -\frac{\Omega-\omega}{2}
\end{array}\right)
$$

and calculate $\mathrm{e}^{-i t B}$ without assuming $\Omega=\omega$ in (25). Again, noting

$$
\begin{aligned}
B^{2} & =\left(\begin{array}{rr}
\left(\frac{\Omega-\omega}{2}\right)^{2}+g^{2} a a^{\dagger} & \left(\frac{\Omega-\omega}{2}\right)^{2}+g^{2} a^{\dagger} a
\end{array}\right) \\
& =\left(\begin{array}{rr}
\left(\frac{\Omega-\omega}{2}\right)^{2}+g^{2} N+g^{2} & \left(\frac{\Omega-\omega}{2}\right)^{2}+g^{2} N
\end{array}\right)
\end{aligned}
$$


$\left(a a^{\dagger}=a^{\dagger} a+1=N+1\right)$ we obtain

$$
\begin{aligned}
\mathrm{e}^{-i t B} & =\exp \left\{-i t\left(\begin{array}{cc}
\frac{\Omega-\omega}{2} & g a \\
g a^{\dagger} & -\frac{\Omega-\omega}{2}
\end{array}\right)\right\} \\
& =\left(\begin{array}{cc}
\cos t \sqrt{\varphi+g^{2}}-\frac{i \delta}{2} \frac{\sin t \sqrt{\varphi+g^{2}}}{\sqrt{\varphi+g^{2}}} & -i g \frac{\sin t \sqrt{\varphi+g^{2}}}{\sqrt{\varphi+g^{2}}} a \\
-i g \frac{\sin t \sqrt{\varphi}}{\sqrt{\varphi}} a^{\dagger} & \cos t \sqrt{\phi}+\frac{i \delta \sin t \sqrt{\varphi}}{\sqrt{\varphi}}
\end{array}\right)
\end{aligned}
$$

where we have set

$$
\delta \equiv \Omega-\omega, \quad \varphi \equiv \frac{\delta^{2}}{4}+g^{2} N
$$

for simplicity. See (26). This is a good exercise for young students. 